\title{
O papel do professor na percepção dos alunos de Odontologia: impacto do ensino de graduação baseado na comunidade
}

Dental students' perceptions and impact role of a community-based educational design for undergraduate education Percepciones de los estudiantes de Odontología y el impacto de enseno universitario basado en la comunidad Carol Grenge Frazille ${ }^{1}$

João Pedro Justino de Oliveira LIMÍRIO ${ }^{1}$ Angelo Camargo DALBEN ${ }^{2}$

Maria Isabel Rosifini Alves REZENDE ${ }^{3}$

Maria Cristina Rosifini ALVES REZENDE ${ }^{\mathbf{1}}$

${ }^{1}$ UNESP - Univ Estadual Paulista Júlio de Mesquita Filho, Faculdade de Odontologia de Araçatuba 16015-050 Araçatuba- SP, Brasil ${ }^{2}$ Defensoria Pública do Estado de São Paulo, Unidade de Araçatuba 16010-030 Araçatuba- SP, Brasil

${ }^{3}$ Programa de Pós-Graduação em Direito, Faculdade de Direito de Ribeirão Preto da Universidade de São Paulo (USP) $14040-906$ Ribeirão Preto - SP, Brasil

\section{Resumo}

O curso de graduação em Odontologia não pode se apartar de seu papel social formador e transformador na construção de um profissional cidadão, reflexivo e que transforme a sua realidade em função das demandas da sociedade. $O$ ensino de graduação que permeie a educação com base na comunidade pode se consubstanciar como ferramenta essencial na construção do olhar sociocomportamental em estudantes universitários, na medida em que promovam a compreensão dos fatores que afetam o bem estar e a qualidade de vida dos indivíduos no cotidiano, tendo o corpo docente como construtor de conhecimentos e fundamentos, gestor de ações planejadas, metódicas e realizadas com determinado objetivo, recriando e superando os modelos de ensino/aprendizagem. A Organização Mundial de Saúde (OMS) recomenda que os currículos de graduação em saúde comportem estratégias pedagógicas capazes de levar à formação de profissionais sensíveis aos problemas de sua comunidade, preparados para a prestação de cuidados em todos os níveis de serviços de saúde. Neste contexto, o propósito deste trabalho foi analisar o papel do professor na percepção dos alunos de Odontologia considerando o impacto do ensino de graduação baseado na comunidade.

Descritores: Aprendizagem; Docentes; Estudantes; Percepção; Universidades; Relações Comunidade-Instituição.

\section{Abstract}

The graduation from a dental school cannot be separated from its formative and transforming social role in the construction of a professional citizen, reflective and transforming its reality according to the demands of society. Undergraduate education that permeates communitybased education can become an essential tool in the construction of the socio-behavioral look in university students, insofar as they promote the understanding of the factors that affect the well-being and quality of life of individuals in their daily lives, having the teaching staff as a builder of knowledge and fundamentals, manager of planned, methodical actions carried out with a determined objective, recreating and surpassing the teaching / learning models. The World Health Organization (WHO) recommends that undergraduate health curricula include pedagogical strategies that can lead to the training of professionals sensitive to the problems of their community, prepared to provide care at all levels of health services. In this context, the purpose of this work was to analyze the role of the teacher in the perception of dental students considering the impact of undergraduate education based on the community.

Descriptors: Learning; Faculty; Students; Perception; Universities; Community-Institutional Relations.

\section{Resumen}

La educación en Odontología no se puede desvincular de su rol social formador y transformador en la construcción de un ciudadano profesional, reflexivo y transformador de su realidad según las demandas de la sociedad. La educación de pregrado que impregna la educación comunitaria puede convertirse en una herramienta fundamental en la construcción de la mirada socioconductual en los estudiantes universitarios, en la medida en que promueva la comprensión de los factores que inciden en el bienestar y la calidad de vida de las personas en su día a día. , teniendo al profesorado como constructor de conocimientos y fundamentos, gestor de acciones planificadas, metódicas y llevadas a cabo con un objetivo determinado, recreando y superando los modelos de enseñanza / aprendizaje. La Organización Mundial de la Salud (OMS) recomienda que los currículos de pregrado en salud incluyan estrategias pedagógicas que puedan conducir a la formación de profesionales sensibles a los problemas de su comunidad, preparados para brindar atención en todos los niveles de los servicios de salud. En este contexto, el propósito de este trabajo fue analizar el rol del docente en la percepción de los estudiantes de odontología considerando el impacto de la educación de pregrado con base en la comunidad.

Descriptores: Aprendizaje; Docentes; Estudiantes; Percepción; Universidades; Relaciones Comunidad-Institución.

\section{INTRODUÇÃO}

A educação baseada na comunidade é ferramenta essencial na construção do olhar sociocomportamental em estudantes universitários da área da saúde, na medida em que promove compreensão dos fatores que afetam o bem estar e a qualidade de vida dos indivíduos no contexto diário $^{1,2}$.

Estudos conduzidos por Dornan et al. ${ }^{1} \mathrm{e}$ Dolman et al. ${ }^{3}$ demonstram que a aprendizagem que coloca o aluno em contato com os determinantes sociais da saúde dos pacientes em sua interface social, econômica, política e social é altamente enriquecedora ao trazer significado ao conhecimento.

Em 1987 a OMS $^{4}$ recomendou que os currículos de graduação em saúde sofressem alterações em suas estratégias pedagógicas capazes de levar à formação de profissionais preparados para o século XXI, sensíveis aos problemas de sua comunidade, voltados para a prestação de cuidados em todos os níveis de serviços de saúde, o que poderia ser alcançado alinhando a educação com as necessidades da comunidade.

$\mathrm{Na}$ Odontologia o aprendizado baseado na comunidade ganha valor adicional quando os estudantes são guiados por um processo reflexivo de aprendizagem $^{5}$, capaz de diminuir a distância entre o pensamento e a ação, entre a teoria e a prática $^{6}$, fomentando a construção de habilidades sociais e humanísticas ${ }^{7}$. Forte et al. ${ }^{8}$ ressalvam a promoção da saúde e a responsabilidade social na formação em saúde como importantes pressupostos na educação em Odontologia baseada na comunidade.

Neste contexto, O Curso de Graduação em Odontologia não pode se apartar de seu papel social formador e transformador na construção de um profissional cidadão, reflexivo e que transforme a sua 
realidade em função das demandas da sociedade. $\mathrm{O}$ ensino de graduação que permeie a educação com base na comunidade pode se consubstanciar como ferramenta essencial na construção do olhar sociocomportamental em estudantes universitários, na medida em que promovam a compreensão dos fatores que afetam o bem estar e a qualidade de vida dos indivíduos no cotidiano, tendo o corpo docente como construtor de conhecimentos e fundamentos, gestor de ações planejadas, metódicas e realizadas com determinado objetivo, recriando e superando os modelos de ensino/aprendizagem ${ }^{9-12}$, Mahler et al. ${ }^{13}$ apontam que o conhecimento e a motivação de um professor são duas características essenciais e incontestáveis para uma educação de sucesso.

Para Hattie ${ }^{14}$ os professores têm mais impacto na aprendizagem dos alunos do que questões ferozmente debatidas na esfera política, como orçamento escolare e tamanho da turma. Neste contexto, o propósito deste trabalho foi analisar o papel do professor na percepção dos alunos de Odontologia considerando o impacto do ensino de graduação baseado na comunidade.

\section{MATERIAL E MÉTODO}

Para a elaboração do presente trabalho as seguintes etapas foram percorridas: estabelecimento da hipótese e objetivos do estudo; estabelecimento de critérios de inclusão e exclusão de artigos (seleção da amostra). Formulou-se a seguinte questão: a percepção do aluno do curso de odontologia com ensino baseado na comunidade sofre influência do professor? Os artigos foram selecionados utilizando a base de dados The National Library of Medicine, Washington DC (MEDLINE - PubMed) e Google Scholar. As estratégias utilizadas para localizar os artigos tiveram como eixo norteador a pergunta e os critérios de inclusão da revisão, previamente estabelecidos para manter a coerência na busca dos artigos e evitar possíveis vieses. Como descritores foram utilizados "Aprendizagem", "Docentes", "Estudantes", "Odontologia", "Percepção", "Universidades" e "Relações ComunidadeInstituição" acordando com o Decs. Os critérios de inclusão foram artigos publicados em inglês, espanhol e português com os resumos disponíveis, no período compreendido entre 2000-2020.

\section{RESULTADOS E DISCUSSÃO}

\section{- Relações Comunidade-Instituição}

Para Walters et al. ${ }^{15}$, apresentar a comunidade aos alunos nos primeiros anos de sua formação profissional parece moldar como eles irão definir suas carreiras e suas responsabilidades éticas.

Estudos de Evans et al. ${ }^{16}$ descreveram o desenvolvimento e a implementação de um currículo de Odontologia com aprendizagem em serviço baseado na comunidade (College of Dentistry, University of Illinois at Chicago). Os autores observaram que além de permitir o aprimoramento do currículo, levar os alunos além dos muros da Universidade permite aos alunos o contato com uma ampla variedade de modelos de prática, de populações de pacientes e de perspectivas sobre sistemas de prestação de cuidados de saúde.

Knight $^{17}$ acrescenta, além da inovação curricular, a inovação de estratégias tradicionais de ensino e aprendizagem. Para Abuzar e Owen ${ }^{18}$ o ensino de graduação baseado na comunidade fornece aos alunos uma ampla gama de experiência clínica e oportunidade de desenvolver o conhecimento das disparidades de saúde bucal entre diferentes comunidades.

Forte et al. $^{8}$ destacam que a educação baseada na comunidade pode ser entendida como uma oportunidade não só do aluno aprender com a comunidade, mas também desenvolver importante etapa do processo de ensino/aprendizagem dentro da comunidade, alicerçando sua formação na responsabilidade social que percebe a comunidade $\mathrm{e}$ seus usuários além das ações acadêmicas, parceiros efetivos e com lugar de fala sobre questões essenciais a respeito de suas condições de saúde e respectivos determinantes.

Segundo Warmling et al. ${ }^{19}$ os novos percursos curriculares da graduação em Odontologia implicam na formação de perfis profissionais predominantemente generalistas voltados para as relações de trabalho e entre o cirurgião-dentista e a sociedade atual no atendimento das demandas para a promoção da saúde bucal.

Donate-Bartfield e Lobb $^{20}$ ressalvam a importância das escolas de Odontologia prepararem os futuros cirurgiões-dentistas para prestarem cuidados culturalmente sensíveis a diversas populações de pacientes, amparados na aprendizagem nas ciências do comportamento, ética e saúde pública.

Araújo e Zilbovicius ${ }^{21}$ apontam a formação acadêmica em Odontologia como forma de preparar cirurgiões-dentistas para "entender e atender" as demandas sociais da população.

- Docentes x Ensino/Aprendizagem

Segundo Aguiar Neta e Alves ${ }^{22}$ novos paradigmas da educação em saúde rompe com o conceito de qualificação dos docentes para ações educativas que preparem os profissionais para atendimento das necessidades da comunidade.

Para Amundsen e Wilson ${ }^{23}$ o desenvolvimento educacional envolve ações planejadas, metódicas e realizadas com determinado objetivo, recriando e superando modelos de ensino/aprendizagem.

Dybowski et al. ${ }^{12}$ estudaram a relação entre a autoeficácia e o entusiasmo dos professores e o desempenho dos alunos por meio de 94 aulas ministradas por 55 professores com 500 avaliações de 
estudantes de um curso de medicina (University Medical Center Hamburg-Eppendorf). Os autores observaram uma relação positiva e significativa entre o desempenho discente e o entusiasmo docente.

Para Ceccim e Ferla ${ }^{24}$ todo ensinar promove cidadania na medida em que fomenta micropercepções em um território ético afetivo e reflexivo.

Mohan e Ravindran ${ }^{25}$ estudaram os fatores que influenciam a preparação do graduando de Odontologia para a prática odontológica e identificaram como relacionados ao treinamento, gênero e experiência dos estudantes/oportunidade de atuação. Para os autores os fatores que influenciam a preparação sugerem que o treinamento odontológico deve refletir os locais de trabalho e as situações reais que os graduandos encontrarão como futuros profissionais.

Holden $^{26}$ pontua que o crescente interesse e reconhecimento da importância do preparo do aluno para a prática odontológica, expõe o aspecto multifacetado desse conceito, estendendo-se além de simples noções de habilidade e competência técnica, incluindo componentes como resiliência, habilidades de comunicação, ideias de profissionalismo e considerações ambientais.

Elmberger et al. $^{27}$ destacam que $\mathrm{o}$ desenvolvimento educacional deve ser exigido dos professores, assegurando-lhes tempo reservado para tais atividades, seja participando de workshops e cursos, seja participando de conferências educacionais ou ainda buscando desenvolvimento educacional no próprio local de trabalho.

Steinert et al. ${ }^{28}$ ressalvam que para alcançar a excelência em ensino e aprendizagem, os membros do corpo docente precisam abraçar suas identidades como professores e serem apoiados para fazê-lo por suas instituições e pelo desenvolvimento do corpo docente. Para os autores a identidade do professor alicerça não só as funções e responsabilidades acadêmicas, como também as oportunidades de desenvolvimento profissional.

- Percepção x Estudante x Ensino/Aprendizagem na Odontologia

Lazzarin et al. ${ }^{29}$ avaliaram as percepções dos professores de Odontologia da Universidade Estadual de Londrina, UEL (Brasil) sobre o papel do professor no processo de ensino e aprendizagem. Seus resultados mostraram que o professor desempenha um papel fundamental no processo de ensino e aprendizagem, sendo o único provedor do conhecimento utilizando estratégias de ensino/aprendizagem baseadas na exposição oral. Grande parte dos professores avaliados obteve sua formação didático-pedagógica a partir de Cursos de Pós-Graduação (Mestrado e / ou Doutorado), que não capacita adequadamente para a carreira docente.
A formação geral, humanista, crítica e reflexiva do aluno é fortemente impactada pela formação e atualização didático-pedagógica do corpo docente.

Seijo et al. $^{30}$ acreditam que incluir as percepções dos alunos no processo educacional é considerado um componente-chave no monitoramento da qualidade dos programas acadêmicos, já que as percepções dos alunos fornecem informações valiosas sobre o desenvolvimento do curso e a relação professoraluno, permitindo aprimoramento do ensino.

Também Victoroff e $\operatorname{Hogan}^{31}$ apontam a visão dos alunos sobre sua experiência educacional como fonte importante de informações para a avaliação do currículo.

Divaris et al. ${ }^{32}$ destacam que a perspectiva do aluno deve ser considerada em todas as discussões e decisões relativas ao ensino da Odontologia com vistas à melhoria da qualidade de vida e o bem-estar dos estudantes, potencializando sua experiência educacional total e influenciando positivamente sua futura carreira. Para os autores, o ambiente acadêmico 'ideal' pode ser definido como aquele que melhor prepara o aluno para a prática odontológica futura, contribuindo para o seu desenvolvimento pessoal, psicossomático e bem-estar social. Fatores diversos influenciam significativamente a maneira como os alunos percebem e vivenciam sua educação; número de alunos por turma, períodos para o lazer, métodos de avaliação da aprendizagem, relação aluno/aluno e aluno/professor, clima ético e oportunidade de atividade extramuro.

Pöhlmann et al. ${ }^{33}$ examinaram a extensão do estresse, esgotamento e problemas de saúde em estudantes de odontologia $(n=161)$ do quarto e quinto ano de três universidades (Dresden, Freiburg e Berna), buscando identificar prevalência de estresse, Burnout e demais problemas de saúde. O estresse foi menor em Berna e consideravelmente maior em Dresden. Diferenças nos níveis médios de sintomas de Burnout foram encontradas apenas para a dimensão exaustão emocional e os alunos de Dresden e Freiburg estavam mais exaustos emocionalmente do que os alunos de Berna; os alunos de Dresden também relataram mais problemas de saúde do que os alunos de Berna ou Freiburg. Dez por cento dos estudantes de Odontologia sofriam de exaustão emocional severa, $17 \%$ reclamaram de uma grave falta de realização e $28 \%$ relataram graves sintomas de despersonalização. Quarenta e quatro por cento da variância da exaustão emocional foi explicada por fatores relacionados ao estudo, como falta de tempo de lazer, ansiedade no exame e estresse de transição. $\mathrm{O}$ único preditor de despersonalização foi a falta de integração social, responsável por $3 \%$ da variância. Para os autores, a falta de integração social pode ser compreendida como um indicador de baixa competência social que pode causar dificuldades em 
lidar com os pacientes de forma adequada e, portanto, resultar em despersonalização. Ademais, os resultados indicam a necessidade de identificar o grupo de alunos que pode ter habilidades sociais insuficientes para lidar adequadamente com os pacientes e treiná-los adequadamente.

Toassi et $\mathrm{al}^{34}$ estudaram a compreensão sobre a percepção do odontolando em relação à vivência da prática em saúde nos serviços de Atenção Básica. Os autores observaram fortalecimento da autonomia, fortalecimento da comunicação e fortalecimento da tomada de decisões do acadêmico, capacitando-o para a compreensão das formas de organização e gestão do trabalho em saúde na comunidade.

Em 2003 Ayers et al. $^{35}$ compararam a percepção de alunos de Odontologia de instituições públicas e privadas sobre a educação baseada na comunidade. Com base nos resultados obtidos os autores defendem as atividades extramuros como componentes importantes de qualquer currículo contemporâneo de Odontologia.

DeCastro et al. ${ }^{36}$ estudaram as perspectivas de ex-alunos sobre currículos de Odontologia egressos de cursos tradicionais $(n=110)$ e egressos de cursos baseados na comunidade $(\mathrm{n}=55)$. Segundo os autores a percepção das demandas da coletividade e o desejo de ajuda-la influenciou as decisões de carreira e prática nos cirurgiões-dentistas graduados nos cursos com formação baseada na comunidade.

Importante destacar que a formação educacional odontológica baseada na comunidade é tão eficaz quanto as experiências educacionais intramuros (formação tradicional) para fornecer aos alunos uma sólida educação clínica, com competência similar ${ }^{37}$.

Neste aspecto Bean et al. $^{38}$ avaliaram a produtividade e as experiências dos alunos do último ano de um curso de Odontologia com formação baseada na comunidade (Ohio State University College of Dentistry, OSUCOD). Os resultados mostraram que os estudantes concluíram em locais da comunidade e em 50\% do tempo tantos procedimentos e equivalentes de receita semelhantes quanto em uma clínica interna (intramuros) da faculdade.

Forte et al. ${ }^{8}$,em seu estudo sobre a percepção dos estudantes de Odontologia de uma instituição pública brasileira sobre sua inserção na comunidade via Sistema Único de Saúde (SUS) e as possíveis contribuições desse processo nas suas formações acadêmicas, observaram que os discentes percebem a comunidade como cenário vivo de aprendizado, capaz de aproximar os serviços de saúde de seus usuários.

Henzi et al. ${ }^{39}$ analisaram quais técnicas de ensino devem ser integradas na prática educacional de rotina pelo corpo docente de odontologia do futuro, e quais aspectos do ambiente de aprendizagem clínica devem ser abordados para melhorar a qualidade geral do experiência para pacientes, alunos e o novo grupo de professores. Para os autores, após o estudo com alunos de Odontologia do primeiro, último ano e pós-graduação em 21 cursos de Odontologia dos Estados Unidos, o aspecto mais forte da educação clínica referido pelos entrevistados foi o relacionamento com o corpo docente, mas também relataram que a clínica da faculdade costumava ser um ambiente de aprendizado ineficiente, atrapalhando suas oportunidades para desenvolver competência clínica. Os alunos também perceberam que a escassez de professores e a crise crescente para a educação odontológica comprometiam seu progresso na clínica e tornavam o aprendizado menos eficiente.

As percepções de 605 estudantes de escolas de Odontologia dos Estados Unidos e Canadá sobre sua educação, considerando os pontos fortes e fracos, oportunidades e ameaças apontaram para preocupações fundamentais sobre a qualidade do ensino em algumas áreas do currículo. Os pontos fortes analisados foram: 1) experiência de aprendizagem clínica e 2) oportunidade de trabalhar com professores experientes. Como pontos fracos: 1) ambiente desorganizado e ineficiente de aprendizagem clínica, 2) ensino e testes que se concentram na memorização, 3) instrução de baixa qualidade caracterizada por desorganização curricular e 4) inconsistência entre professores durante as avaliações dos alunos. As oportunidades da formação se concentraram em: 1) desenvolver estratégias para fornecer aos alunos mais exposição aos pacientes, especialmente no início do currículo, e 2) oportunidades de aprender novas tecnologias e técnicas. Como ameaças à formação foram avaliados: 1) custos da educação odontológica, 2) preocupações dos alunos sobre a "fuga de cérebros" do corpo docente, ou seja, falta de um número suficiente de docentes de odontologia capazes de fornecer instrução de alta qualidade, e 3) tratamento questionável de pacientes na clínica odontológica como consequência da prossecução de requisitos processuais $^{40}$.

Batra et al. ${ }^{41}$ realizaram estudo durante o período do ano letivo 2016/17 com 849 alunos de Odontologia (188 da Croácia, 373 da Índia e 288 do Nepal) objetivando medir as seguintes percepções: flexibilidade, interações aluno-aluno, clima emocional, apoio, experiência significativa, organização e amplitude de interesse. Seus resultados apontaram que os alunos na Croácia avaliaram suas escolas apenas com notas excelentes e boas, enquanto seus colegas na Índia e no Nepal foram mais críticos. Apesar das diferentes configurações em três países, a flexibilidade foi identificada como a área de fraqueza em todos os três sistemas educacionais. 
Em estudo conduzido por Henzi et al. ${ }^{42}$ foram determinadas as percepções dos alunos de Odontologia sobre o ambiente de aprendizagem, o clima intelectual e as relações professor-aluno por meio do instrumento Dental Student Learning Environment Survey (DSLES), que avalia o ambiente de aprendizagem. clima intelectual e relações entre alunos e professores em sete áreas: flexibilidade, interação aluno-aluno, clima emocional, apoio, experiência significativa, organização e amplitude de interesse. O DSLES foi enviado para vinte e três Cursos de Odontologia americanos, com dezoito das escolas distribuindo o inventário e 619 alunos respondentes. Os resultados foram diferenciados entre os alunos calouros e veteranos de Odontologia. Os alunos calouros e veteranos forneceram classificações mais altas (mais positivas) para incentivo ao interesse em Odontologia e interesses externos, além de experiência de aprendizado significativa. Os alunos calouros forneceram as classificações mais baixas para clima emocional (forma como seus cursos foram realizados e níveis de estresse) e os alunos do terceiro ano forneceram as avaliações menos positivas para apoio do corpo docente (grau de apoio do corpo docente e incentivo fornecido aos alunos).

Riquelme et al. ${ }^{43}$ mediram a percepção dos alunos sobre o clima educacional do novo currículo da Pontificia Universidade Católica do Chile utilizando a versão espanhola do Dundee Ready Education Environment Measure (DREEM). Seus resultados apontaram que o clima educacional da escola foi geralmente percebido positivamente pelos alunos, embora eles considerassem o ambiente social da escola de forma menos favorável. As áreas específicas identificadas pelos alunos como necessitando de melhorias incluíam um currículo sobrecarregado e suporte estudantil inadequado.

Mayya e Roff ${ }^{44}$ compararam as percepções sobre o ambiente educacional em alunos com alto e baixo rendimento escolar, buscando a identificação de áreas a serem corrigidas e as diferenças de percepção entre os sexos. Os autores observaram que Mais importância deve ser dada às percepções dos alunos para melhorar o ambiente educacional, uma vez que as percepções estão associadas positivamente com o coeficiente de rendimento, resultado da aprendizagem, abordagem da aprendizagem e atitude em relação ao estudo. As percepções não sofreram influência do sexo do aluno.

Batista et al. ${ }^{45}$ avaliaram a percepção de estudantes de graduação em Odontologia sobre sua capacidade de enfrentar situações de não colaboração na área de Odontopediatria. Um questionário do modelo Likert foi usado para analisar os níveis de autoconfiança dos alunos e soluções propostas para 10 situações-problema que os alunos provavelmente encontrariam na vida profissional. O questionário foi aplicado a dois grupos de alunos de graduação em Odontologia de duas Faculdades Públicas Brasileiras distintas, totalizando 122 respondentes. A análise da autoconfiança indicou que ela variou de acordo com a extensão da reação da criança e a invasividade do procedimento. Os autores concluíram pela importância do treinamento das habilidades de gestão social e comportamental dos alunos de Odontologia.

Freire et al. $^{46}$ acreditam que examinar os perfis e perspectivas dos alunos de Odontologia contribui para as discussões sobre a educação e prática odontológica. Em estudo com 296 calouros brasileiros de uma mesmo Curso de Odontologia matriculados nos períodos 1993-1995 e 2006-2008, por meio de questionário autoaplicável, investigaram a razão da escolha do curso, bem como suas expectativas profissionais. Os resultados apontaram diferenças significativas nas motivações dos alunos calouros e nas perspectivas profissionais ao longo do tempo. A visão pessoal e os conceitos sobre a profissão foram os principais fatores de influência na escolha da Odontologia como carreira. Tendências significativas incluíram um maior interesse na promoção da saúde e serviços públicos, além de especialização em Odontologia Estética e Implantologia no período de 2006-2008.

Ahrheiam et al. ${ }^{47}$ avaliaram as atitudes e a competência percebida entre 108 estudantes de estágio do Departamento de Odontologia Comunitária e Preventiva da Faculdade de Odontologia, de Benghazi, Líbia. Os participantes foram solicitados a fornecer informações demográficas, a responder a afirmações sobre suas atitudes em relação à odontologia preventiva e a responder perguntas sobre sua competência percebida na aplicação de procedimentos odontológicos preventivos. Os resultados mostraram que os aspectos da odontologia preventiva mais reconhecidos foram ser útil e essencial para a comunidade $(95,4$ e $90,8 \%$, respectivamente). $O$ percentual de participantes que expressou proficiência em fornecer orientações de higiene bucal foi o mais alto $(95,4 \%)$. Houve diferenças por gênero e desempenho acadêmico em relação à competência percebida de práticas odontológicas preventivas. Os autores concluíram que o programa de ensino de graduação atualmente implementado na Faculdade de Odontologia de Benghazi (Líbia) não fornece aos dentistas a atitude e as habilidades necessárias para cumprir seu papel na prestação de serviços de saúde com orientação preventiva.

Pinar Erdem et al. $^{48}$ consideram que a educação odontológica desempenha um papel importante em fornecer aos alunos a oportunidade de desenvolver seu conhecimento baseado em evidências e habilidades clínicas, dando suporte aos cuidados preventivos específicos do paciente e às estratégias de gerenciamento de cárie. Em estudo 
examinaram o conhecimento, atitude e competência autopercebida em relação à Odontologia Preventiva entre 126 estudantes do último ano de Odontologia na Turquia, investigando sua prática preventiva para crianças com alto risco de cárie.Os resultados apontaram que $40 \%$ dos estudantes de Odontologia relataram necessidades educacionais e de treinamento quanto ao diagnóstico, agentes preventivos de cárie e plano de tratamento baseado em risco. Para os autores os resultados devem ser levados em consideração pelas partes interessadas no desenvolvimento do currículo básico nacional para o ensino de Odontologia de graduação na Turquia.

Schönwetter et al. $^{49}$ avaliaram as competências de egressos de Odontologia com relação ao impacto das experiências de aprendizagem em sala de aula, clínica e estágios. Os resultados apontaram maior confiança em áreas de procedimentos clínicos básicos envolvendo radiografia, farmacologia e tratamento de cárie, com menor confiança em implantologia, dor orofacial, trauma e tratamento cirúrgico. A maior importância foi atribuída à comunicação interpessoal e habilidades clínicas básicas, com menor importância na pesquisa científica, implantologia e aspectos laboratoriais protéticos. No geral, os egressos sentiram que o ambiente clínico contribuiu mais para o desenvolvimento de competências, seguido pelos contextos de sala de aula e de estágio externo.

Shetty et al. ${ }^{50}$ avaliaram a percepção da experiência de aprendizagem de 45 alunos concluintes de um Curso de Odontologia na Índia. Os domínios avaliados foram currículo de graduação, motivação do aluno e serviços de apoio, infraestrutura institucional, serviços administrativos, componentes de programas de ensino-aprendizagem, nível de confiança na realização de procedimentos clínicos específicos, escolha de carreira e preferência de especialidade de pós-graduação após a graduação. Os resultados apontaram $42 \%$ de concluintes confiantes em relação à prática, com $86 \%$ pretendentes a cursos futuros de especialização. Os autores concluíram que, embora o curriculo tenha sido considerado satisfatório para a maioria dos participantes (95\%), foram identificadas algumas áreas de preocupação que precisam ser melhoradas.

Lanning et al. ${ }^{51}$ analisaram as percepções de 89 alunos (Faculdade de Odontologia da Universidade da Virginia, Estados Unidos) sobre sua experiência educacional com foco no preparo necessário para o atendimento ao paciente e no comprometimento docente com a aprendizagem do aluno. Os resultados apontaram algumas percepções entendidas como comprometedoras da formação: falta de pacientes levou a menor experiência clínica e diminuição na autoconfiança; docentes com habilidades de ensino mostravam baixa interação com os alunos; parte conteúdo curricular foi visto como redundante e irrelevante para a prática futura. Leadbeatter e Peck ${ }^{52}$ destacam que a prática odontológica contemporânea requer profissionais que sejam capazes de recorrer a vários conhecimentos e habilidades interconectados, a fim de fazer julgamentos e agir, quando confrontados com múltiplas, e, muitas vezes contraditórias, maneiras de se interpretar uma mesma situação. Para tanto, os currículos tradicionalmente baseados em conhecimentos teóricos e habilidades técnicas, enquanto instrumentos de formação para a prática odontológica em sociedade, precisam se comprometer como veículo para o desenvolvimento pessoal e profissional, explorando tipos de aprendizagem e abordagens capazes de melhor preparar os graduandos para a atuação futura.

\section{CONSIDERAÇÕES FINAIS}

O Curso de Graduação em Odontologia não pode se apartar de seu papel social formador e transformador na construção de um profissional que transforma a realidade em função das demandas da sociedade. Nesse contexto, o papel docente é fundamental enquanto agente construtor de conhecimentos e fundamentos, gestor de ações planejadas, metódicas e realizadas com objetivo de levar à formação de profissionais preparados para o século XXI, sensíveis aos problemas de sua comunidade, voltados para a prestação de cuidados em todos os níveis de serviços de saúde.

\section{REFERÊNCIAS}

1. Dornan T, Littlewood S, Margolis SA, Scherpbier A, Spencer J, Ypinazar V. How can experience in clinical and community settings contribute to early medical education? A BEME systematic review. Med Teach. 2006;28(1):3-18.

2. Art B, De Roo L, De Maeseneer J. Towards unity for health utilising community-oriented primary care in education and practice. Educ Health (Abingdon). 2007;20(2):74.

3. Dolmans DH, Wolfhagen HA, Scherpbier AJ. From quality assurance to total quality management: how can quality assurance result in continuous improvement in health professions education?. Educ Health (Abingdon). 2003;16(2):210-17.

4. Community-based education of health personnel. Report of a WHO study group [published correction appears in World Health Organ Tech Rep Ser 1987;746:preceding 1]. World Health Organ Tech Rep Ser. 1987;746:1-89.

5. Deogade SC, Naitam D. Reflective learning in community-based dental education. Educ Health (Abingdon). 2016;29(2):119-23.

6. Silverman J, Draper J, Kurtz S. Skills for communicating with patients. Oxon: Radcliffe Medical Press; 2008.

7. Interprofessional education collaborative expert 
panel . Core competencies for interprofessional collaborative practice: report of an expert panel (update) 2016.

8. Forte FDS, Pontes AA, Morais HG, Barbosa AS, Sousa Nétto OB. Olhar discente e a formação em Odontologia: interseções possíveis com a Estratégia Saúde da Família. Interface. 2019;23:e170407.

9. Lestari E, Stalmeijer RE, Widyandana D, Scherpbier A. Understanding attitude of health care professional teachers toward interprofessional health care collaboration and education in a Southeast Asian country. J Multidiscip Healthc. 2018;11:557-71.

10. Roop SA, Pangaro L. Effect of clinical teaching on student performance during a medicine clerkship. Am J Med. 2001;110(3):205-209.

11. Irby DM, Papadakis M. Does good clinical teaching really make a difference. Am J Med. 2001;110:231-32.

12. Dybowski C, Sehner S, Harendza S. Influence of motivation, self-efficacy and situational factors on the teaching quality of clinical educators. BMC Med Educ. 2017;17(1):84.

13. Mahler D, Großschedl J, Harms U. Does motivation matter? - The relationship between teachers' self-efficacy and enthusiasm and students' performance. PLoS One. 2018;13(11):e0207252.

14. Hattie J. Visible learning: A synthesis of over 800 meta-analyses relating to achievement London: Routledge; 2009.

15. Walters L, Greenhill J, Richards J, Ward H, Campbell N, Ash J, et al. Outcomes of longitudinal integrated clinical placements for students, clinicians and society. Med Educ 2012;46:1028-41.

16. Evans CA, Bolden AJ, Hryhorczuk C, Noorullah $\mathrm{K}$. Management of experiences in communitybased dental education. J Dent Educ. 2010;74(10 Suppl):S25-32.

17. Knight GW. Community-based dental education at the University of Illinois at Chicago. J Dent Educ. 2011;75(10 Suppl):S14-S20.

18. Abuzar MA, Owen J. A Community Engaged Dental Curriculum: A Rural Indigenous Outplacement Programme. J Public Health Res. 2016;5(1):668.

19. Warmiling $C M$, Rossoni $E$, Hugo FN, Toassi RFC, Lemos VA, Slavutzki SMB, et al. Estágios curriculares no SUS: experiências da Faculdade de Odontologia da UFGRS. Rev ABENO. 2011; 11(2):63-70.

20. Donate-Bartfield E, Lobb WK, Roucka TM. Teaching culturally sensitive care to dental students: a multidisciplinary approach. J Dent Educ. 2014;78(3):454-64.
21. Araújo ME, Zilbovicius C. O ensino da epidemiologia na educação odontológica. In: Ferreira Antunes JLF, Peres MAP. (Org.). Epidemiologia da saúde bucal. São Paulo: Guanabara Koogan; 2006. p. 363-72.

22. Aguiar Neta A, Alves MSCF. A comunidade como local de protagonismo na integração ensinoserviço e atuação multiprofissional. Trab educ saúde. 2016;14(1):221-35.

23. Amundsen $\mathrm{C}$, Wilson $\mathrm{M}$. Are we asking the right questions? A conceptual review of the educational development literature in higher education. Rev Educa Res. 2012;82(1):90-126.

24. Ceccim RB, Ferla AA. Educação e saúde: ensino e cidadania como travessia de fronteiras. Trab educ saúde. 2008;6(3):443-56.

25. Mohan M, Ravindran TKS. Conceptual Framework Explaining "Preparedness for Practice" of Dental Graduates: A Systematic Review. J Dent Educ. 2018;82(11):1194-202.

26. Holden ACL. "Preparedness for Practice" for Dental Graduates Is a Multifaceted Concept That Extends Beyond Academic and Clinical Skills. J Evid Based Dent Pract. 2020;20(1):101421.

27. Elmberger A, Björck E, Liljedahl M, Nieminen J, Bolander Laksov K. Contradictions in clinical teachers' engagement in educational development: an activity theory analysis. Adv Health Sci Educ Theory Pract. 2019;24(1):125-40.

28. Steinert Y, O'Sullivan PS, Irby DM. Strengthening Teachers' Professional Identities Through Faculty Development. Acad Med. 2019;94(7):963-68.

29. Lazzarin HC, Nakama L, Cordoni Júnior L. Percepção de professores de odontologia no processo de ensino-aprendizagem [Perceptions of dentistry teachers in the teaching and learning process]. Cien Saude Colet. 2010;15 Suppl 1:1801-10.

30. Seijo MO, Ferreira EF, Ribeiro Sobrinho AP, Paiva SM, Martins RC. Learning experience in endodontics: Brazilian students' perceptions. J Dent Educ. 2013;77(5):648-55.

31. Victoroff KZ, Hogan S. Students' perceptions of effective learning experiences in dental school: a qualitative study using a critical incident technique. J Dent Educ. 2006;70(2):124-32.

32. Divaris K, Barlow PJ, Chendea SA, Cheong WS, Dounis A, Dragan IF et al. O ambiente acadêmico: a perspectiva dos alunos. Eur J Dent Educ . 2008; 12 Suppl 1:120-30.

33. Pöhlmann K, Jonas I, Ruf S, Harzer W. Stress, burnout and health in the clinical period of dental education. Eur J Dent Educ. 2005;9(2):78-84.

34. Toassi RFC, Davoglio RS, Lemos VMA. Integração ensino-serviço-comunidade: o estágio na atenção básica da graduação em Odontologia. Educ rev. 2012; 28(4):223-42. 
35. Ayers CS, Abrams RA, McCunniff MD, Goldstein BR. A comparison of private and public dental students' perceptions of extramural programming. J Dent Educ. 2003;67(4):412-17.

36. DeCastro JE, Matheson PB, Panagakos FS, Stewart DC, Feldman CA. Alumni perspectives on community-based and traditional curricula. $\mathrm{J}$ Dent Educ. 2003;67(4):418-26.

37. DeCastro JE, Bolger D, Feldman CA. Clinical competence of graduates of community-based and traditional curricula. J Dent Educ. 2005;69(12):1324-31.

38. Bean CY, Rowland ML, Soller H, et al. Comparing fourth-year dental student productivity and experiences in a dental school with community-based clinical education. J Dent Educ. 2007;71(8):1020-26.

39. Henzi D, Davis E, Jasinevicius R, Hendricson W. North American dental students' perspectives about their clinical education. J Dent Educ. 2006;70(4):361-77.

40. Henzi D, Davis E, Jasinevicius R, Hendricson W. In the students' own words: what are the strengths and weaknesses of the dental school curriculum?. J Dent Educ. 2007;71(5):632-45.

41. Batra M, Ivanišević Malčić A, Shah AF, Sagtani RA, Mikić IM, Knežević PT et al. Self assessment of dental students' perception of learning environment in Croatia, India and Nepal. Acta Stomatol Croat. 2018;52(4):275-85.

42. Henzi D, Davis E, Jasinevicius R, Hendricson W, Cintron L, Isaacs M. Appraisal of the dental school learning environment: the students' view. J Dent Educ. 2005;69(10):1137-47.

43. Riquelme A, Oporto M, Oporto J, Méndez JI, Viviani P, Salech F et al. Measuring students' perceptions of the educational climate of the new curriculum at the Pontificia Universidad Católica de Chile: performance of the Spanish translation of the Dundee Ready Education Environment Measure (DREEM). Educ Health (Abingdon). 2009;22(1):112.

44. Mayya S, Roff S. Students' perceptions of educational environment: a comparison of academic achievers and under-achievers at kasturba medical college, India. Educ Health (Abingdon). 2004;17(3):280-91.

45. Batista CG, Nascimento CL, Rolim GS, Rocha RASS, Rodrigues AF, Ambrosano GMB et al. Student self-confidence in coping with uncooperative behaviours in paediatric dentistry. Eur J Dent Educ. 2011;15(4):199-204.

46. Freire Mdo C, Jordao LM, de Paula Ferreira N, de Fatima Nunes M, Queiroz MG, Leles CR. Motivation towards career choice of Brazilian freshman students in a fifteen-year period. J Dent Educ. 2011;75(1):115-21.
47. Arheiam A, Bankia I, Ingafou M. Perceived competency towards preventive dentistry among dental graduates: the need for curriculum change. Libyan J Med. 2015;10:26666.

48. Pinar Erdem A, Peker K, Kuru S, Sepet E. Evaluation of Final-Year Turkish Dental Students' Knowledge, Attitude, and Self-Perceived Competency towards Preventive Dentistry. Biomed Res Int. 2019;2019:2346061.

49. Schönwetter DJ, Law D, Mazurat R, Sileikyte R, Nazarko O. Assessing graduating dental students' competencies: the impact of classroom, clinic and externships learning experiences. Eur $\mathrm{J}$ Dent Educ. 2011;15(3):142-52.

50. Shetty VB, Shirahatti RV, Pawar P. Students' perceptions of their education on graduation from a dental school in India. J Dent Educ. 2012;76(11):1520-26.

51. Lanning SK, Wetzel AP, Baines MB, Ellen Byrne B. Evaluation of a revised curriculum: a four-year qualitative study of student perceptions. J Dent Educ. 2012;76(10):1323-33.

52. Leadbeatter D, Peck C. Are dental students ready for supercomplex dental practice?. Eur J Dent Educ. 2018;22(1):e116-21.

\section{CONFLITO DE INTERESSES}

Os autores declaram não haver conflitos de interesse.

\section{AUTOR PARA CORRESPONDÊNCIA}

\section{Maria Cristina Rosifini Alves Rezende}

cristina.rosifini@unesp.br

Submetido em 01/05/2020

Aceito em 02/07/2020 